\title{
Characteristics and outcomes of obstetric patients with maternal sepsis requiring admission to a South African intensive care unit: A retrospective review
}

\author{
J Y Lafon, ${ }^{1}$ BSc, MSc, MB BCh, MMed, FCOG (SA); E C Buga, ${ }^{1,2,3}$ MB ChB, Dip Obst (SA), FCOG (SA), Cert Crit Care (SA); \\ G D Nethathe, ${ }^{3} \mathrm{MB}$ ChB, DA (SA), FCA (SA), FANZCA, MMed (Anaes), Cert Crit Care (SA), PGD in HSE \\ ${ }^{1}$ Department of Obstetrics and Gynaecology, Chris Hani Baragwanath Academic Hospital, Johannesburg, South Africa \\ ${ }^{2}$ Department of Intensive Care, Chris Hani Baragwanath Academic Hospital, Johannesburg, South Africa \\ ${ }^{3}$ Division of Critical Care, School of Clinical Medicine, Faculty of Health Sciences, University of the Witwatersrand, Johannesburg, South Africa
}

Corresponding author: J Y Lafon (jayefon@gmail.com)

\begin{abstract}
Background. Maternal sepsis is a major cause of maternal mortality worldwide and constitutes one of the leading causes of preventable maternal mortality and morbidity. Puerperal sepsis following a caesarean delivery has been shown to be a leading cause of sepsis in women.

Objectives. To describe the epidemiology, risk factors, management interventions and maternal outcome of patients with maternal sepsis admitted to a South African (SA) intensive care unit (ICU) over a 2-year period.

Methods. We conducted a single-centre, retrospective review of data of 54 patients admitted to a tertiary referral ICU in SA with the diagnosis of maternal sepsis from 1 January 2015 to 31 December 2016. Clinical characteristics, management interventions and maternal outcomes of patients with maternal sepsis were described and assessed.

Results. There were 39884 deliveries during the study period and 16060 (40.3\%) were by caesarean section (CS). Sepsis was diagnosed in $1.7 \%(n=678)$ of the patients and $8.0 \%(n=54)$ of these patients were admitted to ICU. The median (interquartile range (IQR)) maternal age was $30(26$ - 34) years. Sepsis post CS accounted for 50\% ( $n=27)$, sepsis post vaginal birth for $12.9 \%(n=7)$, septic incomplete miscarriages for $24.0 \%(n=13)$, sepsis post preterm deliveries for $9.2 \%(n=5)$ and sepsis post exploratory laparotomy for ruptured ectopic pregnancy for $3.7 \%(n=2)$. Coagulase-negative Staphylococcus was the most commonly isolated organism. The median (IQR) duration of ICU stay was 4 (2 - 6) days. All patients required mechanical ventilation. The mean (standard deviation (SD)) duration of ventilation was 2.5 (1.7) days. More than a quarter of women $(29.6 \% ; n=16)$ required inotropic support, while $90.7 \%(n=49)$ required blood products and $9.2 \%(n=5)$ required renal replacement therapy. Complications in ICU were metabolic acidosis (46.3\%), acute kidney injury (40.7\%), coagulopathy (25.9\%), circulatory shock (12.9\%), acute respiratory distress syndrome (9.2\%), cardiac arrest (9.2\%) and abnormal liver enzymes (7.4\%). Mortality was $11.1 \%(n=6)$.

Conclusions. Maternal sepsis remains a significant cause of morbidity and mortality. The identification of associated risk factors will help guide appropriate interventions.
\end{abstract}

Keywords. peripartum sepsis; critical illness.

S Afr J Obstet Gynaecol 2020;26(3):89-93. https://doi/org/10.7196/SAJOG.2020.v26i3.1613

Sepsis is a major cause of maternal mortality and morbidity worldwide. Approximately $90 \%$ of all deaths related to pregnancy and childbirth occur in developing countries. ${ }^{[1]}$ Death rates due to sepsis are higher in Africa, Asia, and Latin America and the Caribbean than in developed countries. ${ }^{[2]}$ In the past three decades, efforts have been made by the World Health Organization (WHO) to standardise definitions of sepsis for the general adult population to address the difficulties around terminology in the field. ${ }^{[3]}$ According to the recent surviving sepsis campaign guidelines, sepsis is defined as a life-threatening organ dysfunction caused by a dysregulated host response to infection. Peripartum sepsis is defined by the WHO as bacterial infection of the genital tract or its surrounding tissues occurring at any time between the onset of rupture of membranes or labour and day 42 postpartum in which two or more of the following are present: pelvic pain; fever; abnormal vaginal discharge; abnormal smell/foul odour discharge; or delay in uterine involution. ${ }^{[4]}$
According to the confidential enquiry into maternal deaths, $28.1 \%$ ( $n=100 / 356)$ of women died owing to obstetric haemorrhage, $26.7 \%$ $(n=95 / 356)$ died from hypertension, $12.6 \%(n=45 / 356)$ died from non-pregnancy related infection, 9.6\% $(n=34 / 356)$ died from medical and surgical disorders, $7.6 \%(n=27 / 356)$ died from pregnancyrelated sepsis, $6.7 \%(n=24 / 356)$ died from anaesthetic complications, and $4.5 \%(n=16 / 356)$ died from pulmonary embolism between the periods of 2014 and 2016 in South Africa (SA). ${ }^{[5]}$

Recently, the quick sepsis related organ failure assessment (qSOFA) score or the sepsis 3 consensus definitions have been used to identify patients at risk of sepsis and are at a higher risk of death in the hospital. The qSOFA score creates a standardised, numeric score that allows comparison of patient population groups and helps inform resource allocation and triage decisions. The score uses three criteria and assigns one point for low blood pressure (systolic blood pressure $\leq 100 \mathrm{mmHg}$ ), high respiratory rate ( $\geq 22$ breaths per min) 
or altered mentation (Glasgow Coma Scale $(\mathrm{GCS})<15){ }^{[6]}$

A large number of childbirths and pregnancy terminations still occur outside the healthcare system in developing countries. Additionally, health-seeking behaviour is also poor. ${ }^{[7]}$ Therefore, clinical data on patients presenting to the intensive care units (ICUs) with maternal sepsis are limited in developing countries. We aimed to describe clinical characteristics, management interventions and maternal outcomes of patients with maternal sepsis admitted to a tertiary ICU in SA.

\section{Methods}

The study population included all obstetric patients admitted to Chris Hani Baragwanath Academic Hospital (CHBAH) ICU diagnosed with maternal and peripartum sepsis by the treating intensivist. $\mathrm{CHBAH}$ is a 3200 bed hospital located in Johannesburg, SA, with a maternity unit that delivers an average of 24000 babies every year. Patients whose names were captured in the ICU admission record ledgers and whose files were found in the ICU records room from 1 January 2015 to 31 December 2016 with the clinical diagnosis of maternal sepsis as per the admitting physician were included in the study. Data collected included demographic data, obstetric clinical details, maternal comorbidities and clinical details during ICU stay (Table 1). No files were excluded.

The total number of patients admitted to ICU with maternal and peripartum sepsis during the study period was 54 . The primary objective of the case series review was to determine maternal outcomes as defined by discharge from ICU or mortality of patients admitted with the diagnosis of maternal sepsis to CHBAH ICU. Secondary objectives were to describe the demographic and clinical characteristics of maternal admissions to CHBAH ICU with the diagnosis of maternal sepsis using the qSOFA score to identify risk factors associated with peripartum sepsis and the most common organisms responsible for peripartum sepsis.

Data were entered into a passwordprotected Excel database (Microsoft Corp., USA) and were analysed using the STATA software, version 14 (STATA Corp., USA). Bivariate analyses were performed using Fisher's exact and $\chi^{2}$ tests. The primary outcome variable was mortality. Exposure variables included sociodemographic and clinical data. For descriptive and analytical

Table 1. Demographic and clinical characteristics of patients on admission

\begin{tabular}{|c|c|c|c|}
\hline \multirow[b]{2}{*}{ Characteristic } & \multicolumn{2}{|c|}{ Died, $n(\%)$} & \multirow{2}{*}{$\begin{array}{l}\text { Fisher's exact } \\
p \text {-value }\end{array}$} \\
\hline & Yes & No & \\
\hline \multicolumn{4}{|l|}{ Age, years } \\
\hline$<35$ & $3(7.1)$ & $39(92.9)$ & 1.000 \\
\hline$\geq 35$ & 0 & $9(100)$ & \\
\hline \multicolumn{4}{|c|}{ Premature labour, GA (weeks) } \\
\hline$\leq 28$ & $1(5.9)$ & $16(94.1)$ & 1.000 \\
\hline$>28-37$ & $1(6.7)$ & $14(93.3)$ & \\
\hline$>37$ & $1(5.3)$ & $18(94.7)$ & \\
\hline \multicolumn{4}{|l|}{ Weight (kg) } \\
\hline$<80$ & $1(4.2)$ & $23(95.8)$ & 0.708 \\
\hline $80-100$ & $2(9.1)$ & $20(90.9)$ & \\
\hline$>100$ & 0 & $5(100)$ & \\
\hline \multicolumn{4}{|l|}{ Chronic comorbid disease } \\
\hline No & $3(13.0)$ & $20(86.96)$ & 0.090 \\
\hline Yes & 0 & $27(100)$ & \\
\hline \multicolumn{4}{|c|}{ Prolonged rupture of membranes } \\
\hline No & $2(4.3)$ & $45(95.7)$ & 0.221 \\
\hline Yes & $1(25)$ & $3(75)$ & \\
\hline \multicolumn{4}{|c|}{ Retained products of conception } \\
\hline Uterine evacuation & $3(10.0)$ & $27(90.0)$ & 0.265 \\
\hline No uterine evacuation & 0 & $20(100)$ & \\
\hline \multicolumn{4}{|c|}{ History of pelvic or other infection } \\
\hline Septic miscarriage & $1(4.6)$ & $21(95.5)$ & 1.000 \\
\hline Puerperal sepsis & $2(7.1)$ & $26(92.9)$ & \\
\hline \multicolumn{4}{|l|}{ Diabetes } \\
\hline No & $3(6.1)$ & $46(93.9)$ & 1.000 \\
\hline Yes & 0 & $2(100)$ & \\
\hline \multicolumn{4}{|l|}{ Parity } \\
\hline 1 & 0 & $14(100)$ & 0.383 \\
\hline 2 & $3(14)$ & $17(85)$ & \\
\hline 3 & 0 & $10(100)$ & \\
\hline 4 & 0 & $6(100)$ & \\
\hline \multicolumn{4}{|l|}{ Length of stay in ICU, days } \\
\hline$\leq 6$ & $1(2.4)$ & $40(97.6)$ & 1.000 \\
\hline$>6$ & 0 & $8(100)$ & \\
\hline \multicolumn{4}{|l|}{ Anaemia } \\
\hline No & $1(14.3)$ & $6(85.7)$ & 0.370 \\
\hline Yes & $2(4.7)$ & $41(95.35)$ & \\
\hline \multicolumn{4}{|l|}{ MAP } \\
\hline No & $3(7.3)$ & $38(92.68)$ & 1.000 \\
\hline Yes & 0 & $10(100)$ & \\
\hline \multicolumn{4}{|c|}{ Duration of ventilation, days } \\
\hline$<5$ & $1(2.4)$ & $41(97.6)$ & 1.000 \\
\hline$\geq 5$ & 0 & $7(100)$ & \\
\hline \multicolumn{4}{|l|}{ Renal replacement required } \\
\hline No & $2(7.1)$ & $26(92.9)$ & 1.000 \\
\hline Yes & $1(4.4)$ & $22(95.7)$ & \\
\hline \multicolumn{4}{|l|}{ Culture } \\
\hline No & $1(2.7)$ & $36(97.3)$ & 0.179 \\
\hline Yes & $2(14.3)$ & $12(85.7)$ & \\
\hline
\end{tabular}

purposes, age, weight, parity, gestational age (GA), qSOFA score, admission heart rate, temperature and respiratory rate were categorised to allow for calculations of frequencies and proportions as well as $\chi^{2}$ analysis. The use of inotropic support, the presence of hypotension on admission, chronic comorbid disease, hypertensive disorders of pregnancy, cardiac arrest, acute kidney injury (AKI) and acute respiratory distress syndrome were binary variables. The GCS was recorded on admission and 
on discharge. Data were reported as medians and interquartile ranges due to non-normality.

Ethics approval was obtained from the University of the Witwatersrand Human Research Ethics Committee (ref. no. M170687). This was a retrospective review of data and the requirement for consent was waived by the ethics committee.

\section{Results}

There were 39884 deliveries during the study period and 16060 (40.3\%) were by CS. Of the $1.7 \%(n=678)$ of patients who were diagnosed with sepsis, $8.0 \%(n=54)$ were admitted to ICU.

The median (IQR) maternal age was 30 (26 - 34) years. More than three-quarters of women $(87 \% ; n=46)$ had between 1 and 3 live births, and 13\% $(n=7)$ had 4 previous live births. The mean (standard deviation (SD)) GA at delivery was 30.5 (1.3) weeks.

Half of the patients $(50 \% ; n=27)$ had puerperal sepsis post CS and $12.9 \%(n=7)$ had puerperal sepsis post normal vaginal birth. Two patients were in the first trimester ( $\mathrm{GA}<13$ weeks) while 14 were in the second trimester (GA between 13 and 27 weeks). About a quarter of the patients $(24.0 \% ; n=13)$ had sepsis post incomplete miscarriages, 9.2\% $(n=5)$ had post preterm deliveries and 3.7\% $(n=2)$ had post exploratory laparotomy for ruptured ectopic pregnancy.

Fifty percent of the patients $(n=27)$ were HIV-positive and $51.85 \%(n=14)$ of these patients did not attend antenatal care prior to hospital admission. Moreover, $48.14 \%(n=13)$ of HIV-positive patients were on antiretroviral therapy. Less than a quarter of women (20.37\%; $n=11)$ had pregnancy-related hypertensive disorders. These included gestational hypertension $(n=7)$, defined as high blood pressure $\geq 140 / 90 \mathrm{mmHg}$ after 20 weeks' gestation with no target organ dysfunction, and pre-eclampsia $(n=4)$. One (1.85\%) woman had cardiac disease (peripartum cardiomyopathy) and one (1.85\%) had antiphospholipid syndrome as per laboratory markers and treating physician diagnosis. More than three-quarters of patients $(88 \% ; n=46)$ had hypotension and $19 \%(n=10)$ had hypertension. The majority of the women $(63 \% ; n=34)$ had a normal temperature $\left(36-38^{\circ} \mathrm{C}\right)$ on admission. Less than a tenth $(9 \% ; n=5)$ had acute respiratory distress syndrome. All patients presented with a qSOFA score of at least 2. More than one-third of patients $(42.1 \% ; n=16)$ had a qSOFA of 3 and 2 patients had a qSOFA score of 6. Clinical characteristics as well as the qSOFA scores are shown in Table 2.

All patients required mechanical ventilation. The mean (SD) duration of ventilation was 2.5 (1.7) days. More than a quarter of the patients $(29.6 \% ; n=16)$ required inotropic support, $90.7 \%$

Table 2. Maternal complications and interventions in ICU

\begin{tabular}{lll}
\hline Variable & Description & $\boldsymbol{n}(\%)$ \\
\hline Inotropic support & Yes & $16(30)$ \\
& No & $35(70)$ \\
Cardiac arrest prior to admission & & \\
& Yes & $6(11.1)$ \\
Acute kidney injury & No & $48(88.9)$ \\
& & \\
Acute respiratory distress syndrome & Yes & $32(59.3)$ \\
& No & $22(40.7)$ \\
ICU = intensive care unit. & Yes & $5(9.3)$ \\
& No & $49(90.7)$
\end{tabular}

$(n=49)$ required blood products (packed red cells, fresh frozen plasma, platelets, cryoprecipitate) and $9.2 \%(n=5)$ required renal replacement therapy.

The median and mean (SD) length of stay in the ICU were 5 and 3.9 (1.9) days, respectively.

There were 6 deaths (11.1\%) and $4(67 \%)$ of these were patients referred from local clinics. Half of these patients $(50 \% ; n=3)$ had positive blood cultures and were HIV-positive and another 50\% $(n=3)$ had puerperal sepsis post CS. Table 2 shows the maternal complications and interventions in ICU.

Maternal complications of sepsis were acute kidney injury (40.7\%), coagulopathy (25.9\%), circulatory shock (12.9\%), acute respiratory distress syndrome $(9.2 \%)$, cardiac arrest $(9.2 \%)$ and abnormal liver enzymes (7.4\%). Blood cultures were positive in $25.9 \%$ of cases $(n=14)$.

Table 3 shows the cultured organisms. Coagulase-negative Staphylococcus was the most frequently isolated organism $(n=4)$.

Bivariate analysis by Fisher's exact test was carried out to identify variables with a potential independent effect on mortality. Age (years), premature labour (GA in weeks), weight $(\mathrm{kg})$, chronic comorbid disease, prolonged rupture of membranes, retained products of conception, history of pelvic or other infection, diabetes, parity, length of stay in ICU (days), anaemia, mean arterial pressure, ventilation duration and renal replacement therapy were not statistically significant in predicting mortality.

\section{Discussion}

Maternal deaths from sepsis remains a major cause of death in SA women. In the present study, maternal sepsis was associated with preterm birth and constituted $25 \%$ of sepsis cases admitted to ICU. Maternal sepsis following preterm deliveries occurred postpartum (66.6\%) while one case (1.8\%) was associated with chorioamnionitis. Preterm birth has been associated with increased risk of maternal and neonatal infection. ${ }^{[8]}$

Maternal sepsis was responsible for $5.9 \%$ of ICU admissions during the study period. A study conducted in the Limpopo Province of SA reported a similar finding (6.7\%). ${ }^{[9]}$ Similar studies conducted in other African countries have reported that between 1 and $10 \%$ of ICU admissions were due to obstetric indications. ${ }^{[9-11]}$ In the present study, $11.1 \%$ of obstetric patients admitted to the ICU with sepsis died. This is lower than the mortality rate $(21 \%)$ reported in a study conducted in Durban, SA. ${ }^{[12]}$ The reasons for this could be variable transport or ambulance availability in different regions of the country and lack of specialist obstetrician and critical care.

Table 3. Cultured organisms

\begin{tabular}{ll}
\hline Causative organism & $\boldsymbol{n}(\%)$ \\
\hline Coagulase-negative Staphylococcus & $4(23.3)$ \\
Klebsiella oxytoca & $2(11.8)$ \\
Escherichia coli & $2(11.8)$ \\
Prevotella spp. & $2(11.8)$ \\
Enterococcus faecalis & $2(11.8)$ \\
Group G Streptococcus & $1(5.9)$ \\
Strep. aeruginosa & $1(5.9)$ \\
Staph. aureus & $1(5.9)$ \\
Pseudomonas aeruginosa & $1(5.9)$ \\
Corynebacterium spp. & $1(5.9)$ \\
&
\end{tabular}


The median maternal age was 30 years. Some studies have shown that teenage mothers have an elevated risk of sepsis during labour, while other studies have shown an increased risk of sepsis with maternal age $>39$ years. ${ }^{[13]}$ Age did not have any significant correlation with mortality in the present study.

Multiple studies have shown an increased rate of sepsis post CS at term and sepsis was attributed to emergency CS compared to elective CS. ${ }^{[13,14]}$ Deliveries by CS in the present study were for fetal distress, prolonged second stage of labour, cephalopelvic disproportion, severe pre-eclampsia and haemolysis, elevated liver enzymes, and low platelets syndrome.

Prolonged labour has been identified as a risk factor for sepsis; however, the duration of labour was not taken into consideration in the present study. Of note, $40.7 \%$ ( $n=22$ ) of the patients had 2 or more children. Multiparous women have been shown to be at an increased risk of sepsis and mortality. ${ }^{[15]}$

The majority of patients $(87 \% ; n=48)$ were diagnosed with anaemia (haemoglobin $<10 \mathrm{~g} / \mathrm{dL}$ ) during their stay in ICU. On average, these patients received 2 units or more packed red cells and fresh frozen plasma, while 6 patients received platelets. Anaemia in pregnancy can be physiological and/or attributed to iron, folate and vitamin B12 deficiency, and has a prevalence of $35-75 \%{ }^{[16]}$ The high percentage of anaemia noted in our study could be due to the fact that $43.6 \%(n=24)$ of women did not attend antenatal clinics. Half of the patients (50\%) had retroviral disease, which was associated with anaemia of chronic disease, late presentation at health facilities, lack of compliance with haematinics and haemorrhage secondary to miscarriages.

Half of the women (50\%; $n=27$ ) were HIV-positive and $51.85 \%$ $(n=14)$ of these women did not attend any antenatal clinics and were not on antiretrovirals. SA has a large number of people living with HIV and although deaths owing to non-pregnancy-related infections associated with HIV/AIDS have decreased, HIV remains a major risk factor for sepsis in our setting. ${ }^{[2]}$

All patients had a qSOFA score of 2 or more, suggesting that the score may be a good triage tool for the admission of septic obstetric patients to ICU.

Globally, Gram-negative bacteria are the leading cause of maternal mortality from sepsis. ${ }^{[17]}$ However, the most frequently cultured organism in the present study was coagulase-negative S. aureus (CNS). CNS is relatively indolent organism, which acts as frequent coloniser and may have been a contaminant. ${ }^{[18]}$ Evidence of sepsis and chorioamnionitis following fetoscopy caused by S. epidermidis, a common skin flora, has been described. ${ }^{[19]}$ The finding of a low positive culture rate in the present study could be attributed to multiple factors such as the administration of antibiotics prior to cultures being taken and cultures being taken after the bacteraemic period. Most of the patients received a combination of ampicillin, gentamycin and metronidazole, which were later escalated depending on the culture results and illness progression.

All patients were sedated, intubated and mechanically ventilated on admission. Positive pressure ventilation delivered either noninvasively or invasively is a common therapeutic intervention in this group of patients. ${ }^{[20]}$

More than a third of patients $(44.4 \% ; n=24)$ admitted to the ICU were pyrexial with temperatures above $38^{\circ} \mathrm{C}$. Furthermore, the majority of the patients $(85.2 \% ; n=46)$ had tachycardia (heart rate $>100 \mathrm{bpm}$ ), which could be indicative of sepsis. Tachycardia independent of temperature has previously been shown to be significantly associated with an increased mortality rate. ${ }^{[21]}$
Less than a tenth of the women $(3.7 \% ; n=2)$ were admitted with a preexisting diagnosis of cardiomyopathy, had cardiac arrests in ICU, and were successfully managed with cardiopulmonary resuscitation and ionotropic support. Sepsis-induced cardiomyopathy has been shown to have a mortality rate of $70 \%$ in general cases. ${ }^{[22]}$

More than half of the women $(59.3 \% ; n=32)$ had AKI on admission to ICU. Geraldo et al. ${ }^{[23]}$ showed AKI to be the main complication (24\%) among critically ill obstetric patients admitted with hypertensive syndromes, haemorrhage and haemorrhagic shock, heart disease, respiratory insufficiency and sepsis. These conditions were also associated with increased mortality. ${ }^{[23]}$ The higher rate of AKI in our setting could be attributed to factors such as the presence of pre-existing renal disease, increased comorbidities such as HIV, and poor health-seeking behavior. ${ }^{[23]}$ The majority of the patients $(81.2 \% ; n=26)$ responded to fluid therapy while $11.1 \%$ $(n=6)$ of the patients received renal replacement therapy.

The mortality rate was $10.9 \%(n=6)$ in our study and this was comparable to the reported $11 \%$ in global studies. ${ }^{[24]}$

\section{Study strengths and limitations}

We conducted a small retrospective study in a high-volume $(>20000$ deliveries per year) tertiary academic maternity centre in SA. The present study was limited by its retrospective design. The clinical diagnosis of maternal sepsis was made by the treating physician on admission and may have been subject to clinician variability. Sepsis is diagnosed in a minority of patients in the unit.

Many of the critically ill maternity patients are not admitted to ICU and are managed in the obstetric high-care unit owing to limited resources and a high demand for ICU beds. Therefore, their data was not included in the present study. Furthermore, the hospital is a tertiary referral centre and the population studied may not be representative of the general national group of septic obstetric patients in the community.

\section{Conclusion}

Maternal sepsis remains a major cause of mortality and a major contributor to mortality is puerperal sepsis post CS. The present study describes the characteristics, interventions and outcomes of patients admitted with a diagnosis of maternal sepsis to a tertiary academic ICU in SA. More than half of the women who died from sepsis had preterm delivery and did not attend antenatal clinics. Furthermore, half of the patients in the present study were HIV-positive. CNS was the most commonly cultured organism.

\section{Declaration. None.}

Acknowledgements. We would like to thank Prof. LR Mathivha, Head of Clinical Department at CHBAH for granting approval to conduct this study and Glory Chidumwa for assistance with data analysis and statistical advice.

Author contributions. JYL performed the literature review, collected data, was involved in the analysis and interpretation of data and drafted the manuscript. GDN conceptualised the research project. GDN and ECB supervised the literature review, interpretation of the results, and reviewed the manuscript. All authors approved the manuscript for publication.

Funding. None.

Conflicts of interest. None.

1. World Health Organization. Global Health Observatory (GHO) data - HIV/AIDS. WHO: Geneva, 2018. http://www.who.int/gho/hiv/en/ (accessed 04 January 2018).

2. Moodley J, Pattinson RC, Fawcus S, Schoon MG, Moran N, Schweni PM. The confidential enquiry into maternal deaths in South Africa: A case study. Int J Obstetrics Gynaecol 2014;121(4):53-60. https://doi.org/10.1111/1471-0528.12869 


\section{RESEARCH}

3. Bonet M, Pileggi VN, Rijken MJ, et al. Towards a consensus definition of maternal sepsis: Results of a systematic review and expert consultation. Reprod Health 2017:14(1):67. https://doi. org/10.1186\%2Fs12978-017-0321-6

4. World Health Organization. The prevention and management of puerperal infections: Repor of a technical working group 1992. WHO: Geneva, 1995. https://extranet.who.int/iris/restricted/ bitstream/10665/59429/1/WHO FHE_MSM 95.4.pdf (accessed 14 August 2015).

5. National Department of Health. Saving mothers. South Africa Annual Report on confidential inquiries into maternal death in South Africa. NDoH: Pretoria, 2017. http://www.health gov.za/index.php/shortcodes/2015-03-29-10-42-47/2015-04-30-08-18-10/2015-04-30-08-24-27/ category/559-saving-mothers?download=3414:2017-saving-mothers-annual-report

6. Marik PE, Taeb AM. SIRS, qSOFA and new sepsis definition. J Thorac Dis 2017;9(4):943-945. https://doi.org/10.21037\%2Fjtd.2017.03.125

7. Mhyre JM, Doria R, Hameed AB, et al. The maternal early warning criteria: A proposal from the national partnership for maternal safety. J Obstetric Gynecol Neonatal Nurs 2014;43 (6):771-779. https://doi.org/10.1097/aog.0000000000000480

8. Knowles S, O'Sullivan N, Meenan A, Hanniffy R, Robson M. Maternal sepsis incidence, aetiology and outcome for mother and fetus: A prospective study. Int J Obstet Gynaecol 2015;122(5):663-671. https://doi.org/10.1111/1471-0528.1289

9. Ntuli TS, Ogunbanjo G, Nesengani S, Maboya E, Gibango M. Obstetric intensive care admissions at a tertiary hospital in Limpopo Province, South Africa. South Afr J Crit Care 2015;31(1):8-10. https://doi.org/10.7196\%2FSAJCC.164

10. Okafor UV, Aniebue U. Admission pattern and outcome in critical care obstetric patients. Int J Obstet Anesth 2004;13(3):164-166. https://doi.org/10.1016/j.ijoa.2004.04.002

11. Platteau P, Engelhadt T, Moodley J, Muckart DJ. Obstetric and gynaecological patients in an intensive care unit: A 1-year review. Trop Doctor 1997;27(4):2002-2006. https://doi. org/10.1177/004947559702700406

12. Ngene NC, Moodley J, Songca P. Maternal and fetal outcomes of HIV-infected and noninfected pregnant women admitted to two intensive care units in Pietermaritzburg, South Africa. S Afr Med J 2013;103(8):543-548. https://doi.org/10.7196/samj.6590

13. Conroy K, Koenig AF, Yu Y-H, Courtney A, Lee HJ, Norwitz ER. Infectious morbidity after cesarean delivery: 10 strategies to reduce risk. Reviews Obs Gynecol 2012;5 (2):69-76.
14. Seo K, McGregor JA, French JI. Preterm birth is associated with increased risk of maternal and neonatal infection. Obs Gynecol 1992;79(1):75-80.

15. Allen VM, Baskett TF, O'Connell CM, McKeen D, Allen AC. Maternal and perinatal outcomes with increasing duration of the second stage of labor. Obs Gynecol 2009;113(6):1248-1258. https://doi org/10.1097/aog.0b013e3181a722d6

16. Vincent JL, Baron J-F, Reinhart K, et al. Anemia and blood transfusion in critically ill patients. J Am Med Assoc 2002;288(12):1499-1507. https://doi.org/10.1001/jama.288.12.1499

17. Duan R, Xu X, Wang X, Yu H. Perinatal outcome in women with bacterial sepsis: A cross-sectional study from West China. Medicine (Baltimore) 2019;98(44):e17751. https://doi.org/10.1097/ MD. 0000000000017751

18. Bone RC. Gram-negative sepsis: A dilemma of modern medicine. Clin Microbiol Rev 1993;6(1):57 68. https://doi.org/10.1128/cmr.6.1.57

19. Levin G, Zigron R. Case report of Staphylococcus epidermidis maternal sepsis and chorioamnionitis following fetoscopy. Int J Gynaecol Obstet 2018;140(2):253-254. https://doi.org/10.1002/ijgo.12359

20. Tapaan P, Sunita S, Sasmita B. Obstetric admissions to the intensive care unit: A five-year review. Int J Reprod Contracept Obstet Gynecol 2015;4(6):1914-1917. https://doi.org/10.18203/2320-1770. ijrcog20151285

21. Leibovici L, Gafter-Gvili A, Paul M, et al. Relative tachycardia in patients with sepsis: An independent risk factor for mortality. Int J Med 2007;100(10):629-634. https://doi.org/10.1093/ qjmed/hcm074

22. Liu YC, Yu MM, Shou ST, Chai YF. Sepsis-induced cardiomyopathy: Mechanisms and treatments. Front Immunol 2017;8(3):3-5. https://doi.org/10.3389\%2Ffimmu.2017.01021

23. Silva Junior GBD, Saintrain SV, Castelo GC, et al. Acute kidney injury in critical ill obstetric patients: A cross-sectional study in an intensive care unit in Northeast Brazil. Braz J Nephrol 2017;39(4):357 361. https://doi:10.5935/0101-28000.20170066

24. Romero-Bermejo JF, Ruiz-Bailen M, Gil-Cebrian J, J Huertos-Ranchal M. Sepsisinduced cardiomyopathy. Curr Cardiol Rev 2011;7(3):163-183. https://doi.org/10.2174\% 2F157340311798220494

Accepted 23 March 2021 